\title{
TAGUNG
}

\section{Administrative Reformen in internationalen und supranationalen Organisationen}

\author{
Michael W. Bauer und Helen Foerster*
}

Beim Regieren im Zeitalter von Globalisierung und Europäisierung spielen internationale und supranationale Organisationen (nachfolgend abgekürzt als IOs) eine immer wichtigere Rolle. Immerhin gibt es weltweit mittlerweile mehr als 5000 öffentliche, also auf der Zusammenarbeit von nationalen Regierungen beruhende Organisationen, die auf einer formalen Übereinkunft basieren, mehr als drei Staaten als Mitglieder aufweisen und darüber hinaus auch über ein permanentes Sekretariat - eine Verwaltung - verfügen. Angesichts der aktuellen Bedeutung von IOs erstaunt es, dass den internen Reformprozessen dieser ,transnationalen Administrationen' bisher kaum systematische Aufmerksamkeit geschenkt wurde. Nun konnte bis vor wenigen Jahren mit einigem Recht argumentiert werden, dass es in diesem überstaatlichen Verwaltungsbereich eben nicht gerade viel Modernisierungsgeschehen gebe. Gerade in jüngster Zeit haben aber viele wichtige IOs - so zum Beispiel die Europäische Kommission, das Europäische Parlament (EP), die Europäische Zentralbank (EZB), die OECD und einige Unterorganisationen der Vereinten Nationen - ihre internen Strukturen und Verwaltungsabläufe sowie ihr Personal- und Finanzmanagement zum Teil grundlegend erneuert. Was wissen wir über diese Prozesse und wie lässt sich das beobachtbare Reformgeschehen einordnen?

\section{Die Reform der Europäischen Kommission}

Noch am besten erforscht sind die jüngsten Verwaltungsreformen der Europäischen Kom-

\section{Management Reforms in Internatio- nal and Supranational Organisations}

Internationale Tagung des Arbeitskreises Europäische Integration (AEI) in Zusammenarbeit mit dem Lehrstuhl für Vergleichende Policy-Forschung und Verwaltungswissenschaft am Fachbereich Politik und Verwaltung der Universität Konstanz

Konstanz, 30. Juni bis 1. Juli 2006

\section{Wissenschaftliche Leitung:}

Dr. Michael W. BAUER, Universität Konstanz

\section{Welcome and Introduction}

Prof. Dr. Brigitte ROCKSTROH, Vice-President of the University of Konstanz

\section{Reforming the European Commission}

Chair: Prof. Dr. Christoph KNILL, University of Konstanz

Against the Grain? The Reform of the European Commission

Prof. Hussein KASSIM, Birkbeck, University of London

Reforming ethics in the European Commission: from the 2000 White Paper to the Transparency Initiative

Prof. Michelle CINI, University of Bristol

Deserving to be made ,Sir"? - Effects of the Reform of the European Commission on Policy Output

Dr. Michael W. BAUER, University of Konstanz

\section{Comments}

Emmanuelle SCHÖN-QUINLIVAN, University College Cork

* Dr. Michael W. Bauer, wissenschaftlicher Assistent, Lehrstuhl für Vergleichende Policy-Forschung und Verwaltungswissenschaft, Universität Konstanz.

Helen Foerster, wissenschaftliche Mitarbeiterin, Lehrstuhl für Vergleichende Policy-Forschung und Verwaltungswissenschaft, Universität Konstanz. 
mission. Das Interesse von Michelle Cini gilt dabei dem Stellenwert der , public ethics', der Entwicklung ethischer Standards in Politik und Verwaltung (Einstellung zu Betrug, Korruption und Verschwendung) im Reformprozess dieser Institution. Die Bedeutsamkeit der ,öffentlichen Ethik' ermisst sich schon daraus, dass hier zentrale Fragen des Vertrauens der Bevölkerung in die Kommission und damit der Legitimation der europäischen Integration insgesamt berührt werden. Cini argumentierte, dass Fragen der Ethik zwar zu den ersten auf der Reform-Agenda gezählt hätten, im eigentlichen Reformprozess zwischen 1999 und 2004 aber ins Hintertreffen geraten und erst jüngst mit der (unausgegorenen) Initiative für mehr Transparenz wieder aufgegriffen worden seien. Hussein Kassim beschäftigte sich in seinem Vortrag vor allem mit den ,großen Rätseln', die die Reform der Kommission seiner Meinung nach aufwirft: Die Durchführung einer derartig radikalen Reform widerspreche allen theoretischen Erklärungen von Reformwahrscheinlichkeit. Design und Inhalt der Reform, die die Kommission als ,Agent" selbst gestaltet und durchgesetzt habe, gingen weit über das vom ,Prinzipal', also den Mitgliedstaaten, erteilte Mandat hinaus. Nach Kassim leiden Reformen von IOs unter dem strukturellen Problem, dass sich die Organisationen nicht selbstständig, ohne Intervention des Prinzipals, reformieren können. Dessen Interesse sei jedoch beschränkt, weswegen er nur im Falle einer Krise interveniere. Dennoch könne sich die Organisation Mängeln durchaus bewusst sein und eine interne ReformAgenda entwickeln. Es gebe Belege dafür, dass die politische Führung und das leitende Management der Kommission ein solches Bewusstsein im Jahr 1999 entwickelt habe. Die durch den Rücktritt der Santer Kommission verursachte Krise und das daraus resultierende Interesse des Prinzipals öffneten ein , window of opportunity. "Nun konnten Reformen durchgeführt werden, auch solche, die über das Mandat des Prinzipals hinausreichten. $M i$ chael W. Bauer fragte in seinem Vortrag nach den Auswirkungen der Reform der Kommission. Hat die Reform die Kapazität der Kom-
Reforming the European Parliament and other European Organisations

Chair: Dr. Michael W. BAUER

Administrative Reforms in the European Parliament Tarvo KUNGLA, European Parliament

The restructuring process of the Nordic Council of Ministers (NCM) and the Council of the Baltic Sea States (CBSS)

Tobias ETZOLD, Manchester Metropolitan University

Explaining Personnel Reforms in International Organisations: Experiences from OECD and the European Commission

Prof. Dr. Christoph KNILL/Tim BALINT, University of Konstanz

Comments

Stephan GROHS, University of Konstanz

Reforming the United Nations, the World Bank and other International Organisations Chair: Tarvo KUNGLA

The current Reform Initiatives at the United Nations - observations from a Special Envoy

Karl Th. PASCHKE, Special Envoy of the German Government for UN Reform, New York

Factors affecting United Nations Accountabilities: between managerial reform and social claims Daniele ALESANI, Bocconi University, Milan New decision-making procedures inside the World Bank: How International Organisations regain governance capability

Michael KERLER, University of Bamberg

Politics of Management Reforms in International Organisations: The Reform Broker Concept Barbara KOBLER/Soo Mee BAUMANN/Markus HAGEL, University of Konstanz

Comments

Dr. Dominik BÖLLHOFF, Federal Chancellery, Berlin

\section{Roundtable}

Chair: Dr. Dominik BÖLLHOFF

Maria MAGUIRE, Head of Policy Development \& Diversity, $O E C D$

Achim von HEYNITZ, Senior Adviser FAO/ IFAD

Gabriel GLÖCKLER, Counsellor to the Vice-President, European Central Bank

Miguel FIGUEROLA, Director, Human Resources Division, WTO 
mission, Gesetzesvorlagen zu entwerfen, einzubringen und durchzusetzen, verändert? Die Ergebnisse einer standardisierten Befragung von Kommissionsbeamten legten nahe, dass die Reform durch die Transformation der Rolle der Referatsleiter einen unbeabsichtigten Nebeneffekt erzielt habe. Die Referatsleiter bildeten bislang das Rückgrat der Kommission in Sachen Politikgenerierung - im Sinne des Konzepts des politischen Unternehmertums. Sie entwickelten (normalerweise) die Ideen und setzten sich im Getriebe der Kommission und in den Verhandlungen auf der Arbeitsebene mit den anderen Institutionen sowie mit gesellschaftlichen Akteuren für deren Verwirklichung ein. Nach der jüngsten Kommissionsreform sei die Rolle der Referatsleiter jedoch eine reine Managementfunktion geworden. Das Resultat sei daher sehr wahrscheinlich ,better managers, poorer entrepreneurs'. Damit seien aus, integrationsfreundlicher" Perspektive negative Effekte auf die Politikgestaltungsfähigkeit der Kommission zu erwarten.

Interne Reformen im Europäischen Parlament, im Nordischen Rat und in der OECD

Tarvo Kungla referierte über die jüngsten Reformen im Europäischen Parlament. Durchgeführt worden seien vor allem organisatorische (etwa Umstrukturierung von Generaldirektionen, Umsiedlung dieser von Luxemburg nach Brüssel), aber auch Verwaltungsreformen (etwa Einrichtung neuer Projektgruppen). Erklärt werden könnten diese Reformen vor allem mit Umfeldveränderungen (sukzessive Ausweitung der Befugnisse des EP, Anstieg der Beschäftigtenzahlen in Folge von EU-Erweiterungen, Reform der Beschäftigungsvorschriften aller EU-Institutionen, Reform der finanziellen Vorschriften). Diese Umfeldreformen hätten ein ,window of opportunity geöffnet, das zentrale Akteure innerhalb des EPs nutzen konnten. Tobias Etzold analysierte die Reformprozesse im Nordischen Rat (NCM) und im Ostseerat (CBSS). Im Unterschied zu den zuvor analysierten Reformfällen handele es sich hierbei um sehr kleine Organi-

\author{
Looking Ahead \\ Chair: Dr. Michael W. BAUER \\ Innovation and Effectiveness in European Public \\ Management: Practical Needs and Theoretical \\ Possibilities \\ Professor Les METCALFE, Bocconi University, \\ Milan /University of Durham \\ Administrative Reforms as a Means of Improving \\ the Legitimacy of International Organisations \\ PD Dr. Veith MEHDE, University of Hamburg \\ Comments \\ Prof. Dr. Wolfgang SEIBEL, University of Kon- \\ stanz \\ Wrap Up: Prospects of Reforming Internatio- \\ nal Organisations \\ Dr. Michael W. BAUER, University of Kon- \\ stanz
}

sationen mit ausschließlich nordeuropäischen Mitgliedstaaten. Wie haben sie auf Veränderungen in ihrem Umfeld (Zusammenbruch der Sowjetunion, EU-Nord- und Osterweiterung) reagiert? Zusammenfassend diagnostizierte Etzold sowohl beim NCM als auch beim CBSS eine Anpassung im Sinne einer Optimierung vorhandener Strukturen und Prozesse - eine große Reform sei nicht festzustellen. Der Vergleich der beiden Organisationen werfe allerdings neue Fragen auf: Welche Rolle spielt die Größe einer Organisation, welchen Einfluss hat die Zusammensetzung der Mitglieder, wie wichtig sind die Höhe des Budgets und die damit verbundenen Interessen der Mitglieder? Schließlich stellten Christoph Knill und Tim Balint den Stand ihrer Forschung zu Personalreformen in der OECD und der Europäischen Kommission vor. Beide Organisationen seien daraufhin untersucht worden, ob sie in den Jahren 1996 und 2006 auf bestimmten Indikatoren eher dem Bürokratiemodell Webers oder dem Konzept des New Public Management (NPM) entsprachen. Der Vergleich habe ergeben, dass sich die OECD zwischen 1996 und 2006 eher vom NPM-Modell weg bewegte, also ihren selbst propagierten Ansprüchen an modernes Verwaltungspersonalmanagement im öffentlichen 
Sektor selbst immer weniger gerecht werde. Dagegen habe die als inflexibel geltende Kommission weitgehende personalpolitische Reformen verwirklicht. Im Bereich des Trainings sei die Kommission 2006 beispielsweise viel weiter vom Weberschen Bürokratiemodell entfernt als es noch 1996 der Fall war. Von Interesse sei auch, dass die einschlägige Organisationswandeltheorie des institutionellen Isomorphismus genau das Gegenteil nämlich weiter gehende Reformen in der OECD als in der Kommission - erwarten lasse. Eine mögliche alternative Erklärung könne der Grad der Sicherheit sein, die eine Organisation im Hinblick auf ihre Zukunft habe. Denn bei der OECD sei die, Überlebensunsicherheit" weitaus größer als bei der Kommission. Vor dem Hintergrund existenzieller Überlebensängste gemäß der Frage ,Was ist eigentlich der Sinn und Zweck der Organisation?' würden administrative Optimierungsprozesse zu einer Nebensache mit vergleichsweise geringem Stellenwert. Anders als man also auf der Grundlage des Isomorphismus erwarte, führe nur mittlere Unsicherheit zu organisatorischer Angleichung, extreme Unsicherheit jedoch zur Stagnation.

\section{Die administrativen Reformen der Vereinten Nationen und der Weltbank}

Von dem Reformprozess innerhalb der UN berichtete Botschafter Karl Th. Paschke. Er konstatierte einen außerordentlichen Reformbedarf, um Effizienz und Effektivität zu steigern, verwies jedoch auf die politische Realität: Eine Reform erfordere die Zustimmung von 191 Regierungen, hinzu komme der Nord-Süd-Konflikt. Dieser Antagonismus zwischen den Staaten des armen Südens und denen des reichen Nordens habe auch das Schicksal der jüngsten Reforminitiative Kofi Annans stark beeinflusst. Insbesondere Konflikte im für Budgetverhandlungen zuständigen ,5. Komitee ' zwischen USA und Japan auf der einen Seite und Staaten der Dritten Welt auf der anderen hätten eine wirkliche Verwaltungsreform verhindert. Die USA und Japan wünschten eine andere Organisation, die EU wünsche eine effizientere und effektivere, und die Mitglieder der G77 seien der Überzeugung, dass jede Veränderung eine Verschlechterung darstelle. Daniele Alesani analysierte die ,accountability", also die Rechenschaftsablegungsstrategien, von UN-Organisationen. Er identifizierte die wesentlichen Faktoren, die Einfluss auf die Eigenschaften der externen Rechenschaftsablegung haben könnten: organisatorische Faktoren, Faktoren betreffend ,corporate governance' sowie externe Faktoren. Nach Alesani kann jedoch kein einschlägiger Theorieansatz den jeweiligen Umgang der Organisationen mit Rechenschaftspflichten umfassend erklären. Rechenschaftsablegung, so der Verdacht, werde von den Organisatoren eher als, Schaufensterveranstaltung' gesehen und wesentlich nachlässiger behandelt als im privaten Sektor üblich. Im Anschluss daran referierte Michael Kerler über die Reform von Entscheidungsverfahren in der Weltbank. In Bezugnahme auf Niklas Luhmann argumentierte Kerler, dass es in Fällen, in denen institutionelle Subsysteme über sehr viel Autonomie verfügen, zu Dysfunktionalitäten kommen könne. Dies sei in den 1980er Jahren in der Weltbank geschehen. 1991 habe der ,Wopenhans Report' diese Mängel aufgedeckt und somit ,externen Druck' erzeugt, in dessen Folge es dann auch zu umfassenden Reformen gekommen sei. Schließlich stellten Barbara Kobler, Soo Mee Baumann und Markus Hagel eine Arbeit über die Wichtigkeit der Generalsekretäre für Managementreformen in IOs vor. In den fünf von der Arbeitsgruppe untersuchten Fällen, in denen weitreichende Reformen durchgeführt worden waren, hätten die Generalsekretäre auch tatsächlich hohes, commitment' zu den Reformen gezeigt. In den fünf Fällen, in denen keine größeren Verwaltungsreformen (in den ausgewählten IOs) stattgefunden hätten, habe nachgewiesen werden können, dass der Generalsekretär auch nur wenig für die Durch- und Umsetzung von Reformen getan habe. Der Generalsekretär sei also von auBerordentlicher Bedeutung für den Umfang und den Erfolg von Verwaltungsreformen der IOs. 
Eine Podiumsdiskussion mit hochrangigen Managern von IOs

Bei der Podiumsdiskussion kamen die Praktiker ausführlich zu Wort. Jeder einzelne stellte zunächst kurz den Reformprozess in seiner eigenen Organisation vor. Diskutiert wurde die Rolle der Mitgliedstaaten als Reformpromotoren. Gemeinhin gehe der entscheidende Reformdruck tatsächlich von den Regierungen der Organisationsmitglieder aus. Eine Ausnahme stelle hier allerdings die Europäische Zentralbank dar. Gabriel Glöckler führte aus, dass die EZB bis 2002 eine konkrete Aufgabe - die Euro-Einführung - zu bewältigen gehabt habe. Erst im Anschluss daran sei deutlich geworden, dass das Aufgabenwachstum eine Anpassung der administrativen Strukturen erforderlich mache. Der Reformimpuls sei dabei jedoch aus der EZB selbst gekommen. Anschließend wurde noch einmal die Frage aufgegriffen, ob es gemeinsame Merkmale der Reformdefizite und -prozesse gebe. Man einigte sich darauf, dass die Organisationen ähnliche Probleme hätten, wobei allerdings der Dringlichkeitsgrad variiere. Besonders schwierig gestalte sich der Aufbau von Vertrauen zwischen den - zahlreichen - externen wie internen ,Stakeholdern' der jeweiligen IO. Hierzu mangele es häufig an der erforderlichen Managementkapazität. Die kulturelle Dimension und die sich daraus ergebenden Schwierigkeiten spielten in allen Organisationen eine zentrale Rolle. Schließlich könne es als gemeinsames Merkmal der vorhandenen Reformdefizite betrachtet werden, dass IOs normalerweise hoch spezialisiert seien und ihre Mitarbeiter gemeinhin aufgrund individueller technischer Expertise und nicht wegen ihrer Managementqualitäten auswählten. Daraus ergebe sich ein struktureller Mangel an guten Managern in den IOs.

\section{IO-Reform zwischen Kapazitätsdefiziten und Legitimationsnöten}

Im abschließenden Panel stellte zunächst Veith Mehde seine Überlegungen zu Verwaltungsreformen und Legitimationsoptimie- rung von IOs vor. IOs benötigten Legitimation, da sie nicht nur regulative und administrative Funktionen ausübten, sondern auch Entscheidungen träfen, die möglicherweise negative Konsequenzen für Staaten oder Individuen mit sich brächten. Nun seien aber sowohl das Konzept der Input- als auch der Output-Legitimation im Bezug auf diese Organisationen problematisch: Erstere führe zu einer sehr langen ,Legitimationskette', letztere lasse sich vor allem deshalb nicht gut anwenden, da nur wenig direkter Kontakt mit der Bevölkerung bestehe. Wie könne also die Legitimation verbessert werden? Mehde verwies auf Reformen auf nationaler Ebene, die oft symbolischen, die Legitimation stärkenden Zwecken dienten, wie beispielsweise stärkere Serviceorientierung. Mögliche Strategien für IOs seien zum Beispiel Public RelationsInitiativen sowie die Veränderung von Rechenschaftsstrukturen. Schließlich diene auch eine Effizienzsteigerung diesem Zweck. Daher könnten auch administrative Reformen in diesem eingeschränkten Sinne dazu beitragen, Output-Legitimitation zu steigern. Les Metcalfe referierte schließlich über eine Theorie der Effektivität. Effektivität sei eine Funktion von Komplexität (z.B. neue Aufgaben) und Kapazität. Steige die Komplexität, wie zum Beispiel durch die Erweiterung der EU, müsse auch die Kapazität steigen, wie beispielsweise die Managementkapazitäten. Metcalfe nannte zwei Beispiele, die belegen, warum die EU - bei Zugrundelegung dieser Theorie - nicht effektiv handeln könne: Der Lissabon-Prozess, in dessen Rahmen der Kommission neue Aufgaben übertragen worden seien ohne ihre Kompetenzen zu erweitern, sowie die Wirtschafts- und Währungsunion. In beiden Bereichen müssten Netzwerke enormer Größe und Heterogenität koordiniert werden. Dazu bedürfe es inter-organisatorischer Netzwerkkapazitäten, die schlichtweg in der Kommission nicht vorhanden seien.

\section{Fazit}

Insgesamt hat die Tagung - so der Konsens in der abschließenden Diskussion - den Fokus 
auf Phänomene der Reform von IOs gelegt, die bislang kaum systematisch untersucht worden sind. Angesichts der steigenden Bedeutung der ,transnationalen Verwaltungen " für sozioökonomische, sicherheits- und handelspolitische Bereiche modernen Regierens diesseits und jenseits des Nationalstaates scheint es dringend geboten, dem Wandel des administrativen Unterbaus der IOs weiter nachzuspüren. Dabei ist - insbesondere mit Blick auf die Institutionen der Europäischen Union - klar, dass Verwaltungsreformen nicht nur Effektivität und Effizienz steigern müssen, sondern eines der wenigen Mittel darstellen, das Vertrauen der Bürger in Strukturen der Zusammenarbeit im über- und zwischenstaatlichen Raum herzustellen beziehungsweise zu erhalten. Gerade für IOs gilt in viel stärkerem Maße als für nationale Verwaltungen, dass die Akzeptanz bei der Bevölkerung leicht verspielt aber schwer wieder herzustellen ist. Gleichzeitig fehlen den IOs die normalen parteipolitischen und gesellschaftlichen ,Transmissionsriemen', um interne Veränderungen anzustoßen und über einen längeren Zeitraum zu begleiten. Die Erforschung der Faktoren und Bedingungen erfolgreicher interner Reformen von IOs könnte gerade hier einen wichtigen Beitrag leisten.

\section{Schriften zur Europäischen Integration und Internationalen Wirtschaftsordnung}

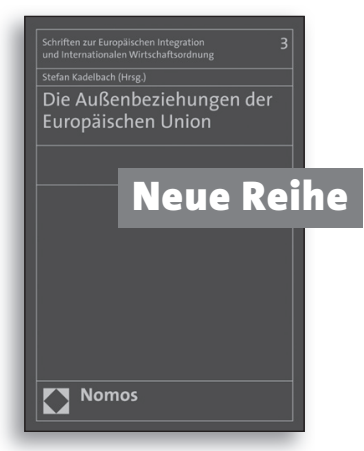

\section{Die Außenbeziehungen \\ der Europäischen Union}

Herausgegeben von Stefan Kadelbach

2006, Band 3, 183 S., brosch., 38,- €, ISBN 3-8329-2337-3

Aus interdisziplinärer Sicht erörtern Experten die globale Rolle der EU mit ihren Widersprüchen und ihrer zunehmenden Reichweite: vom Handel im Rahmen der WTO über die Entwicklungszusammenarbeit hinzur Gemeinsamen Außen- und Sicherheitspolitik. Als regionale Schwerpunkte analysiert der Band die Beziehungen der EU zum Westbalkan und zu China und Südostasien.
Die Konvention der Vereinten Nationen zur Bekämpfung der Korruption

Betrachtungen aus Wissenschaft und Praxis

Herausgegeben von Rainer Hofmann und Christina Pfaff, LL.M.

2006, Band 2, 95 S., brosch., 22,- $€$ ISBN 3-8329-2238-5

\section{Europäische Verfassung und direkte Demokratie Herausgegeben von Stefan Kadelbach 2005, Band 1, 110 S., brosch., 24,- $€$ ISBN 3-8329-1704-7}

